\title{
基于小波包能量曲率差法的桥梁损伤识别试验研究
}

\author{
余 竹 $^{1,2}$, 夏 乐 $^{1}$, Goicolea $\mathrm{J} \mathrm{M}^{2}$, 战家旺 ${ }^{1}$ \\ (1. 北京交通大学 土木建筑工程学院, 北京 100044 ;2. Technical University of Madrid, Madrid 28040)
}

\begin{abstract}
摘 要：针对各种损伤识别指标中,小波包能量曲率差法仅有数值仿真、未经试验验证,尤其缺少实际工程验证问 题, 用数值模拟验证该方法识别结构损伤的有效性; 利用沧州子牙河新桥替换下的梁体, 进行两种工况损伤模拟。通过测 试完好与损伤状态各点加速度响应, 用小波包能量曲率差法识别损伤, 考察小波函数和分解层数对识别效果的影响。结 果表明,该方法有效,并可应用于实际工程。
\end{abstract}

关键词: 小波包分析;小波包能量曲率差;损伤模拟;损伤识别;试验

中图分类号: 0327

文献标识码: A

\section{Experimental study on bridge damage identification based on wavelet packet energy curvature difference method YU Zhu ${ }^{1,2}$, XIA $\mathrm{He}^{1}$, GOICOLEA J $\mathrm{M}^{2}$, ZHAN Jia-wang ${ }^{1}$}

(1. School of Civil Engineering, Beijing Jiaotong University, Beijing 100044, China;

2. Technical University of Madrid, Madrid 28040, Spain)

Abstract: Among various damage identification methods, the Wavelet Packet Energy Curvature Difference (WPECD) method is an effective one. As a regret, most of the existing methods rely only on numerical simulation and are unverified via experiment, and moreover very few of them has been applied to practice. In the paper, the validity of WPECD in structural damage identification was verified by a numerical example. A damage simulation experiment was taken on a real replaced girder at the Ziya River New Bridge in Cangzhou. Two damage cases were simulated and the acceleration responses at the measuring points were obtained, based on which the damages were identified with the WPECD method, and the influences of wavelet function and decomposition level were studied. The results show that the WPECD method can identify structure damage efficiently and can be put into practice.

Key words : wavelet packet analysis; WPECD; damage simulation; damage identification; experiment

结构在服役期间,关键部位会不断累积损伤与缺 陷, 影响其使用安全。因此, 结构健康监测对工程结构 使用性能的评估具有重要意义。结构健康监测可划分 为局部和整体检测两种。局部损伤检测采用红外线探 伤、超声波等技术获取损伤信息; 整体检测通过结构振 动特性变化评价结构健康情况 ${ }^{[1]}$ 。传统基于结构振动 的损伤识别方法多通过对瞬态信号的 Fourier 变换得到 结构模态参数, 如频率、振型 ${ }^{[2-3]}$ 。然而损伤为典型的 适于高频模态表征的局部现象, 而 Fourier 变换对高频 模态分析不足,变换过程中时域信息会丢失,对损伤特 性评价会不准确。

小波变换为克服其它信号处理技术缺点的信号分

基金项目：国家自然科学基金(51078029); 国家重点基础研究计划 973 项目 (2013CB036203); 中央高校基本科研业务费专项资金资助项目 (2012JBM080)

收稿日期: 2012-02-15 修改稿收到日期:2012-03-26

第一作者 余 竹 男,博士生, 1985 年 11 月生

通讯作者 夏 禾 男,教授, 博士生导师,1951 年 4 月生
析方法。小波由一簇基函数构成, 可描述信号时间 (空 间)和频率(尺度) 域的局部特性。采用小波分析的最 大优点是可对信号实施局部分析,可在任意时间或空 间域中分析信号。小波分析能发现其它信号分析方法 不能识别的、隐藏于数据之中的表现结构特性的信息, 而这些特性对损伤识别尤为重要。因此, 小波变换已 成为结构损伤识别中常用方法, 并有大量研究成 果 $^{[4-5]}$ 。然而, 小波变换在高频区域内分辨率仍较低， 在识别含接近高频部分信号时存在一定困难。小波包 变换弥补了小波变换缺陷, 对小波空间进行分解,使正 交小波变换中随尺度的增大而变宽的频谱窗口进一步 分割变细,可提供完整的、不同水平下的分解。利用小 波包理论进行损伤识别成为研究热点。丁幼亮等 ${ }^{[6-7]}$ 在理论与数值上利用小波包能量谱对结构损伤进行预 警及试验验证。Sun 等 ${ }^{[9]}$ 对结构响应进行小波包分析, 用小波包信号能量作为神经网络输人, 以此识别结构 损伤。韩建刚等 ${ }^{[10]}$ 提出基于小波包变换的能量变化率 
指标识别结构损伤,并进行试验验证。

在已有的基于小波包进行结构损伤识别研究中， 大多处于理论研究阶段, 只有少量的试验验证。然而, 实际情况中的桥梁损伤相对实验室条件更复杂多变。 为此, 本文提出基于小波包能量曲率差指标, 用数值与 现场实桥试验, 验证该方法识别结构损伤的有效性,并 研究不同小波函数及小波包分解层数对损伤识别的 影响。

\section{1 基于小波包分析的损伤识别方法}

\section{1 小波包理论介绍}

小波变换具有多分辨率特点,在时频域都具有表 征信号局部特征的能力。虽然小波分解的各层分辨率 不同,但每层所取子带固定, 仅对低频部分进行分解, 故其也有高频段分辨率差的缺点, 因此只适用一定特性 信号。而小波包分析则将每层所有子带均一分为二,并 传至下一层, 对高频和低频进行分解。每层子带都覆盖 原信号所占频率,只是各层分辨率不同,如图 1 所示。

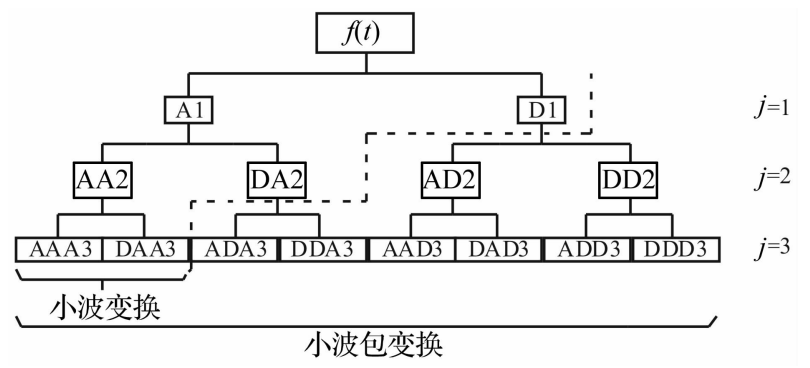

图 1 小波变换与小波包变换

Fig. 1 Wavelet transform and wavelet packet transform

小波包分析对信号分析更精确, 可对多分辨率分 析中未细分的高频部分进一步分解, 可根据被分析信 号特征,选择相应频带使之与信号频谱相匹配, 从而提 高时频分辨率。

小波包函数通常用 $\psi_{j, k}^{i}$ 表示, $i, j, k$ 分别表示小波 包函数的调制、尺度、平移参数,其表达式为:

$$
\psi_{j, k}^{i}(t)=2^{j / 2} \psi^{i}\left(2^{j} t-k\right) \quad(i=1,2, \cdots)
$$

小波函数 $\psi^{i}$ 递推关系式为:

$$
\begin{aligned}
\psi^{2 i}(t) & =\sqrt{2} \sum_{-\infty}^{+\infty} h(k) \psi^{i}(2 t-k) \\
\psi^{2 i+1}(t) & =\sqrt{2} \sum_{-\infty}^{+\infty} g(k) \psi^{i}(2 t-k)
\end{aligned}
$$

式中: $\psi$ 为小波母函数, $h(k), g(k)$ 为与尺度函数及小 波母函数相关的积分镜像滤波器系数。对任意信号的 第 $j$ 阶和第 $j+1$ 阶水平小波包分解递推关系为:

$$
\begin{gathered}
f_{j}^{i}(t)=f_{j+1}^{2 i-1}(t)+f_{j+1}^{2 i}(t) \\
f_{j+1}^{2 i-1}(t)=H f_{j}^{i}(t) \\
f_{j+1}^{2 i}(t)=G f_{j}^{i}(t)
\end{gathered}
$$

式中: $H, G$ 分别对应 $h(k), g(k)$ 的滤波算子, 表达
式为:

$$
\begin{aligned}
& H\{\cdot\}=\sum_{k=-\infty}^{\infty} h(k-2 t) \\
& G\{\cdot\}=\sum_{-\infty}^{\infty} g(k-2 t)
\end{aligned}
$$

经 $j$ 水平的小波包分解后,初始信号 $f(t)$ 表示为:

$$
f(t)=\sum_{i=1}^{2 i} f_{j}^{i}(t)
$$

小波包组分信号 $f_{j}^{i}(t)$ 可表示为小波包函数的线性 组合:

$$
f_{j}^{i}(t)=\sum_{-\infty}^{+\infty} c_{j, k}^{i}(t) \psi_{j, k}^{i}(t)
$$

小波包系数计算式为:

$$
c_{j, k}^{i}=\int_{-\infty}^{+\infty} f(t) \psi_{j, k}^{i}(t) \mathrm{d} t
$$

其中,小波系数满足正交性条件:

$$
\psi_{j, k}^{m}(t) \psi_{j, k}^{n}(t)=0 \text { 当 } m \neq n
$$

小波包分解常用在能量监测中。小波包信号能量 定义为:

$$
E_{f}=\int_{-\infty}^{\infty} f^{2}(t) \mathrm{d} t=\sum_{m=1}^{2^{i}} \sum_{n=1}^{2 j} \int_{-\infty}^{\infty} f_{j}^{m}(t) f_{j}^{n}(t) \mathrm{d} t
$$

将式 (10) 代人式 (13), 并利用正交条件式 (12) , 得:

$$
E_{f}=\sum_{i=1}^{2^{j}} E_{f_{j}^{i}}
$$

式中: 小波包组分能量 $E_{f_{j}}$ 可视为存储于组分信号 $f_{j}^{i}(t)$ 的能量:

$$
E_{f_{j}^{i}}=\int_{-\infty}^{\infty} f_{j}^{i}(t)^{2} \mathrm{~d} t
$$

式中: $E_{f}$ 为信号 $f(t)$ 的能量。

式 (14) 可解释为信号的总能量由对应不同频带内 的小波包组分能量之和。

组分能量对信号特性变化十分敏感,可用于揭示 信号的固有特征。假如结构发生损伤,其响应信号的 某段频率会发生波动, 按小波包分解的信号分量中某 个或某几个分量必会发生能量的增减。可通过测量各 频段信号能量分布的变化诊断结构损伤。

\section{2 不等间距曲率求解方法}

在实际中,曲率一般由变量的二阶差分 (即斜率的 变化率)得到,如图 2 所示。

不等间距情况下曲率求解式为:

$$
K_{i}=y_{i}^{*}=\frac{\frac{y_{i+1}-y_{i}}{h_{i+1}}-\frac{y_{i}-y_{i-1}}{h_{i-1}}}{\frac{h_{i+1}+h_{i-1}}{2}}
$$

式中:分子部分为节点左右两段曲线斜率差, 分母为节 点左右两端斜率差间距。若节点等间距, 即 $h_{i-1}=$ $h_{i+1}$, 则: 


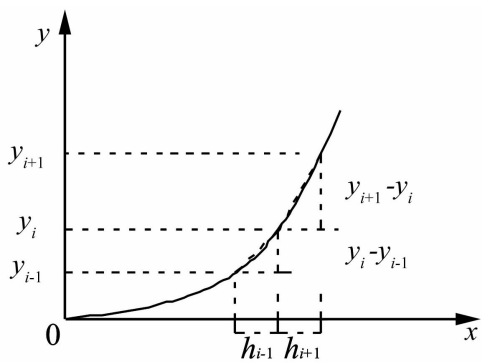

图 2 曲率示意图

Fig. 2 Curvature sketch

$K_{i}=y_{i}^{*}=\frac{\frac{y_{i+1}-y_{i}}{h}-\frac{y_{i}-y_{i-1}}{h}}{h}=\frac{y_{i+1}-2 y_{i}+y_{i-1}}{h^{2}}$

式 (17) 即为二阶差分法求解等间距曲率公式。

将式 (16) 中 $y$ 换成小波包能量 $E_{f_{j}^{i}}$, 即为小波包能 量曲率。将完好状态与损伤状态时各节点的小波包能 量曲率进行插值即得损伤状态的小波包能量曲率差:

$$
\Delta K_{i}=K_{i}^{u}-K_{i}^{d}
$$

式中: $K_{i}^{u}, K_{i}^{d}$ 分别为完好状态与损伤状态的小波包能 量曲率。本文将用该指标进行结构损伤识别。

\section{2 数值算例}

以图 3 简支梁为例, 梁长 $20 \mathrm{~m}, E=1 \times 10^{8} \mathrm{~Pa}, \rho=$ $2500 \mathrm{~kg} / \mathrm{m}^{3}, A=1 \mathrm{~m}^{2}, I=0.083 \mathrm{~m}^{4}$ 。共划分 20 个单 元, 21 个节点, 在梁中点施加 $100 \mathrm{~N}$ 的激振力。采用 ANSYS 进行瞬态分析, 用刚度下降模拟损伤, 假定单元 $5 、 13$ (对应节点号 5-6,13-14) 的刚度分别下降 5\%, $10 \%$ 。对损伤前后 21 个节点在激振力作用下的位移 响应进行小波包变换, 小波函数为 Daubechies 小波, 序 号 $N=15$, 即 Db15 小波, 分解层数为 7 层。前 8 个分量 的小波包能量曲率差如图 4 所示。

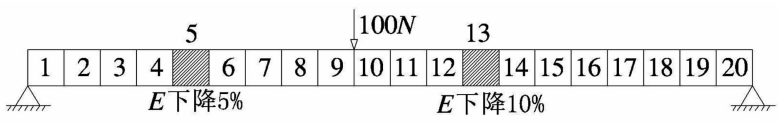

图 3 损伤简支梁示意图

Fig. 3 Sketch of the damaged girder
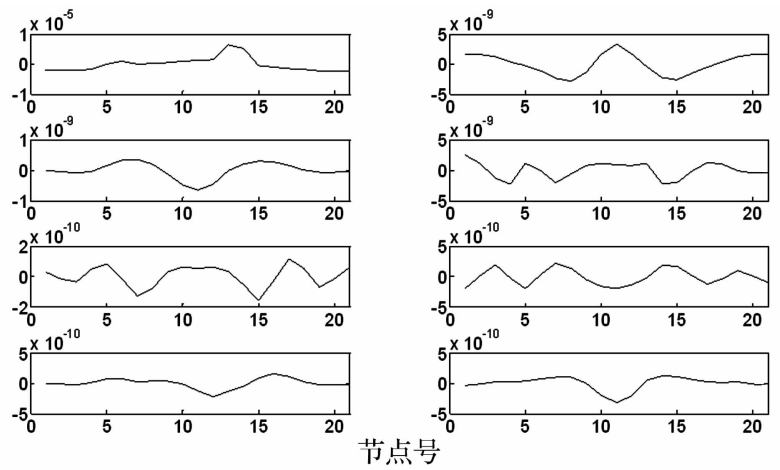

图 4 损伤简支梁小波包信号能量曲率差 (前 8 个分量)

Fig. 4 WPECD of damaged girder (First 8 components)

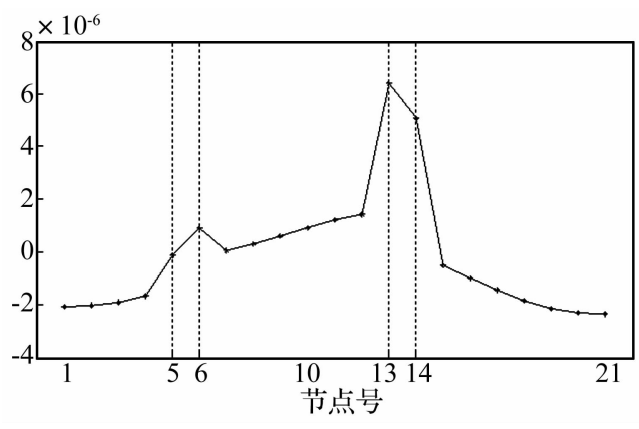

图 5 小波包信号能量曲率差叠加 (前 8 个分量)

Fig. 5 Sum of the first 8 WPECD components

由图 4 看出,第 1 个能量分量的数量级 $\left(10^{-5}\right)$ 较 其余的 $\left(10^{-9}, 10^{-10}\right)$ 大得多,将前 8 个分量的能量叠加 如图 5 所示。

由图 5 可见,结构损伤处能量曲率差有相应的突 变,损伤程度较小的单元 5(损伤 5\%) 也突变明显。说 明该方法对损伤程度较敏感。

考察测点稀疏对识别结果影响, 选节点 $1,3,5, \cdots$, 21 共 11 个测点,损伤识别结果如图 6 所示, 单元 5 处 $5 \%$ 的损伤信息被淹没,而单元 13 处 $10 \%$ 的损伤仍被 识别。可见测点过于稀疏对较小程度损伤影响较大。 因此在实际测试中, 对可能发生损伤的部位, 测点尽可 能密集。

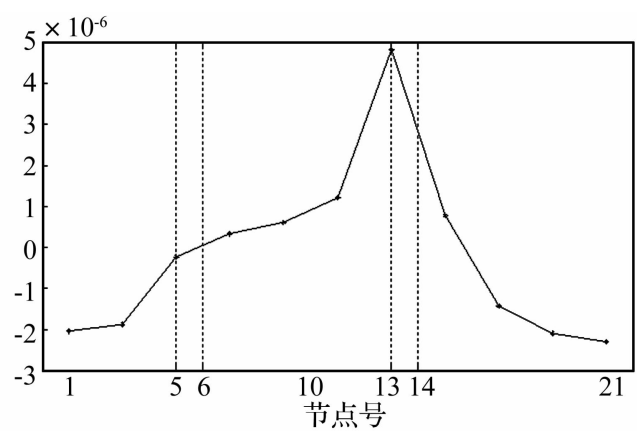

图 6 稀疏测点下小波包信号能量曲率差叠加 Fig. 6 WPECD under sparse measuring points

\section{3 试验研究}

\section{1 试验简介}

以沧州市 205 国道子牙新河上一片简支梁为试验 对象, 如图 7 所示。该梁长 $12.06 \mathrm{~m}$ (两端支座间距), 宽 $1 \mathrm{~m}$ 。在梁纵中心线上布置 19 个测点, 跨中附近 (损 伤位置) 测点布置相对较密。对梁体采用力锤激励 (图 8 ), 敲击 14,15 节点间中间位置。为使每个工况激励 力大小相同, 将力锤置于相同高度处自由下落。

\section{2 损伤工况模拟}

按计划,损伤分两步进行:

(1) 丵除跨中处底部 $10 \mathrm{~cm}$ 厚硂、切断西侧梁底 2 排钢筋, 此时梁体无裂纹。损伤位置为图 7(b) 中 10 号 


\section{节点。}

(2) 切断跨中处底面钢筋, 仅留 2 根, 此时梁体出 现通体裂纹,下端裂纹宽 $3 \mathrm{~mm}$ 。

对损伤状态 (2), 虽切割跨中位置钢筋, 但裂纹却 发生在距跨中偏南 $25 \mathrm{~cm}$ 处 (图 7(b) 中 9 号节点), 如 图 9。实际损伤位置应位于节点 9,10 之间, 且节点 9 损伤会更大。

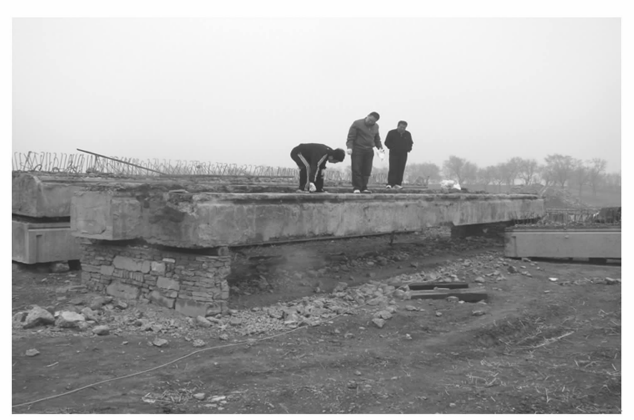

(a) 试验梁

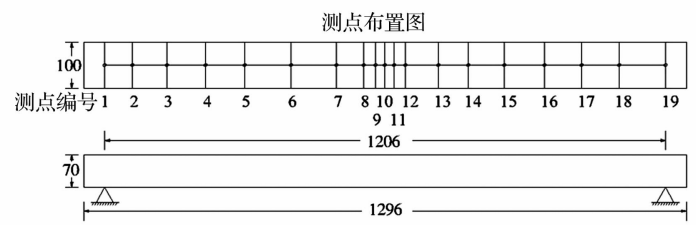

(b) 测点布置 (上: 俯视图, 下: 立面图)

图 7 试验梁及测点布置

Fig. 7 Sketch and measurement arrangement of the girder
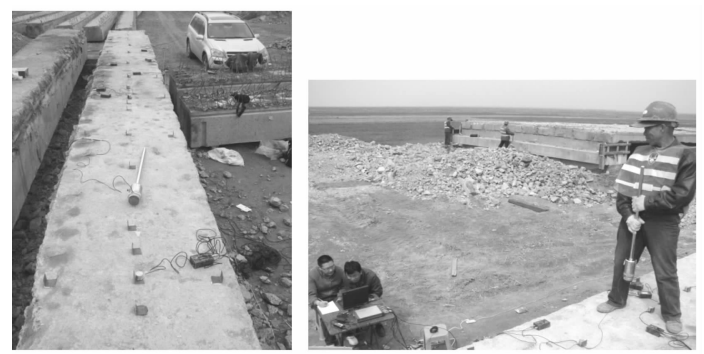

图 8 力锤激励

Fig. 8 Excitation of hammer
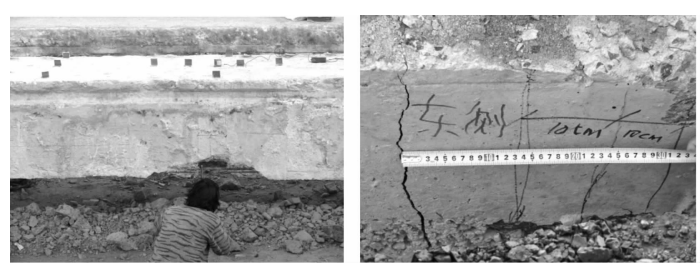

图 9 损伤工况 (左: 损伤 1 , 右: 损伤 2)

Fig. 9 Damage case (L: Case 1, R: Case 2)

\section{3 振动测试}

梁完好状态及两种损伤状态下, 测得 19 个测点加 速度响应。以 14 节点为例, 在损伤工况 (1)下的加速 度时程如图 10 所示。对三种工况下的加速度响应进 行频谱分析,得到梁的自振频率如表 1 所示。

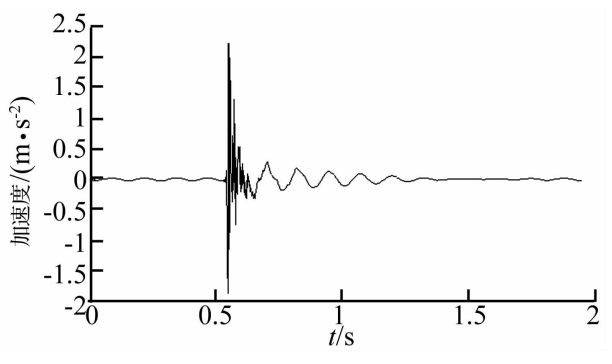

图 10 实测加速度时程 (14 节点, 损伤工况 1)

Fig. 10 Measured acceleration time-history

表 1 完好与损伤工况下梁一阶频率及变化率

Tab. 1 The $1^{\text {st }}$ order frequency and variation of the girder

\begin{tabular}{cccc}
\hline 工况 & 一阶频率/ $\mathrm{Hz}$ & 一阶频率变化率 $V^{*}$ & 损伤位置 \\
\hline 完好状态 & 8.704 & - & - \\
损伤工况 (1) & 8.192 & $-5.88 \%$ & 10 号节点 \\
损伤工况 (2) & 6.144 & $-29.4 \%$ & 9,10 号节点 \\
\hline
\end{tabular}
频率

注: $V^{*}=\left(f_{d}-f_{u}\right) / f_{u}$, 其中 $f_{d} f_{u}$ 分别为损伤状态和完好状态

可据自振频率降低判断结构损伤的发生。降低越 多, 说明结构损伤程度越大。但损伤位置的识别不可 忽略。

\section{4 基于小波包能量曲率差的损伤识别}

将每个节点在三种工况下的响应进行小波包变 换, 分解层数为 7 层, 小波函数为 Db15 小波, 得 $2^{7}=$ 128 个小波包系数分量及能量, 2 种损伤状态下小波包 信号能量曲率差前 8 个分量的叠加见图 11 。

由图 11 可见,损伤 1 工况的小波包信号能量曲率 差在节点 10 处突变较大, 损伤位置明显; 损伤工况 2 , 最大突变在节点 9 处,而实际裂缝也位于该处,表明小 波包能量曲率差对损伤位置具有良好的识别效果。

\section{4 .1 小波函数影响}

考察小波函数对识别结果影响, 采用 Coif5 小波, 分解层数仍取 7 层。损伤识别结果如图 12 所示。

与图 11 相比看出, 2 种小波包函数计算结果差别 很小,均能有效识别结构损伤。计算时间基本相同, Db15 小波和 Coif5 小波函数耗时分别为 $50 \mathrm{~s}$ 和 $48 \mathrm{~s}$ 。 可见,两种小波函数的识别效果和计算效率相差不大， 均可作为小波包能量曲率差识别损伤的方法, 且可相 互验证。

\section{4 .2 小波包分解层数影响}

考察小波包分解层数对识别结果影响,一般,小波 包分解层数越高, 识别效果越好, 但耗时也越长。仍采 用 Db15 小波,分解层数取 8 层。损伤识别结果如图 13 所示。

与图 11 相比, 峰值稍有减小, 但二者识别结果一 致。计算耗时 $101 \mathrm{~s}$, 接近分解 7 层的 2 倍, 由于分解层 
数每增加一层, 小波包信号分量增加 1 倍, 计算时间也 必然增加。因此, 需综合考虑小波包分解层数影响, 选 择合适的分解层数,在保证识别质量的前提下尽可能 减少计算时间。本例中,分解层数 7 层已满足要求。

\subsection{3 小波包能量曲率差损伤识别方法总结}

由以上分析, 基于小波包能量曲率差识别损伤的 步骤总结如下:

（1）结构未损伤前, 测出结构完好状态下节点加
速度响应;

（2）监测服役结构相同节点加速度响应;

(3) 选择合适的小波函数及分解层数, 对各工况 下的节点响应进行小波包分解;

（4）计算各节点的小波包能量曲率差, 只选前几 阶小波包系数能量较大的分量, 观察结果, 有较大突变 处可能发生损伤, 应引起关注, 并及时检查相应节点附 近的健康状况。

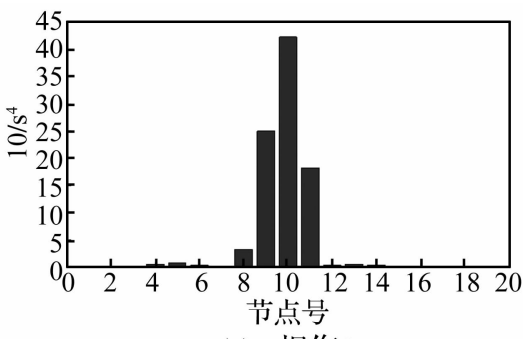

(a) 损伤 1

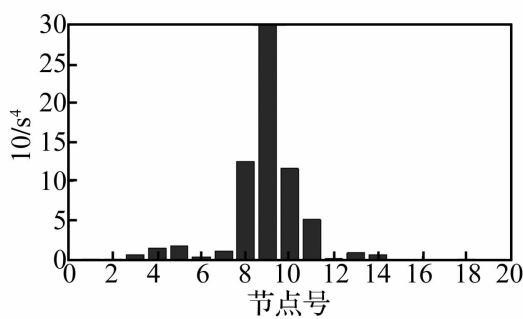

(b) 损伤 $2 \mathrm{db} 15$

图 11 小波包信号能量曲率差 Fig. 11 WPECD

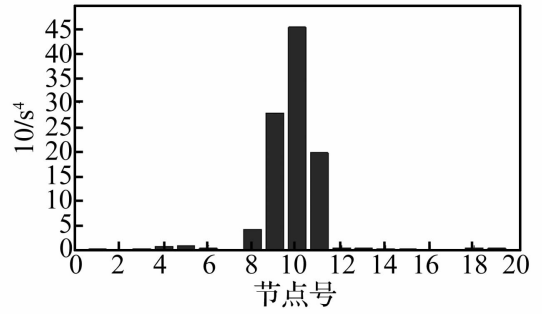

(a) 损伤 1

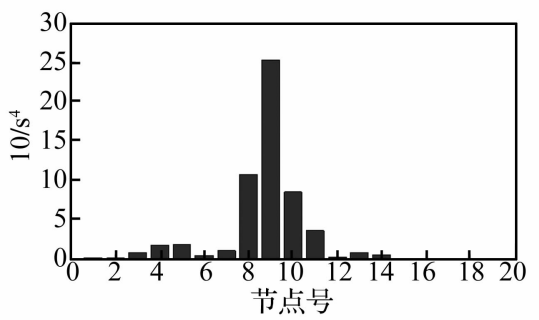

(b) 损伤 2 coif 5

图 12 小波包信号能量曲率差 Fig. 12 WPECD

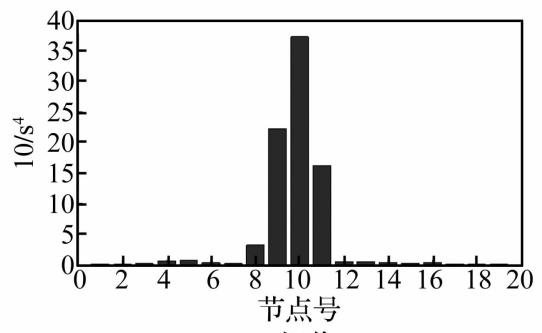

(a) 损伤 1

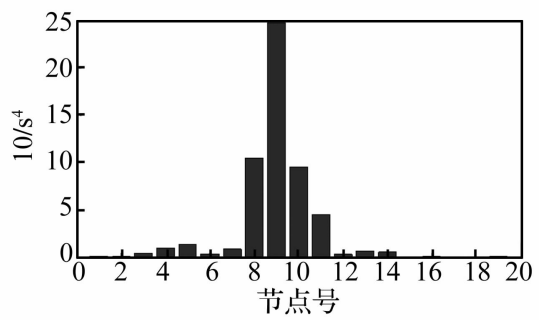

(b) 损伤 $2 \mathrm{db} 15$, 分解层数 8

图 13 小波包信号能量曲率差 Fig. 13 WPECD

\section{4 结 论}

本文通过对一简支梁进行损伤的数值仿真分析表 明,小波包能量曲率差可有效识别损伤位置。并利用 实桥损伤实验验证该方法的有效性。结论如下：

(1) 数值计算表明小波包能量曲率差法对损伤较 敏感, 即是微小损伤 (5\%)，也能有效识别。但测点过 于稀疏会淹没较小程度的损伤信息。

(2) 通过实桥实验验证该方法可有效应用于实 际,为工程结构健康在线监测提供了参考。

（3）不同小波函数对识别效果和计算效率影响不 大,可用不同小波函数分别识别,以相互验证。

（4）小波包分解层数越多,耗时越多。需综合考 虑识别效果与计算效率。

\section{参 考 文 献}

[ 1 ] Doebling S W , Farrar C R, Prime M B. A summary review of vibration-based damage identification methods $[\mathrm{J}]$. Shock Vib. Dig. , 1998, 30(2) :91 - 105 .

[2] Farrar C R, Jauregui D A. Comparative study of damage identification algorithms applied to a bridge. I . experiment [J]. Smart Mater. Struct. , 1998,7:704 - 719.

[ 3 ] Farrar C R, Jauregui D A. Comparative study of damage identification algorithms applied to a bridge. II . numerical study $[J]$. Smart Mater. Struct, 1998, 7:720 - 731.

[ 4 ] Hou Z, Noori M, St. Amand R. Wavelet-based approach for structural damage detection $[\mathrm{J}]$. Eng. Mech. Div. , ASCE, $2000,12(7): 677-683$

[5] Kitada Y. Identification of nonlinear structural dynamics systems using wavelets [J]. Eng. Mech. Div., 1998, 124 (10): $1059-1066$.

[ 6 ] 丁幼亮,李爱群,缪长青. 基于小波包能量谱的结构损伤 预警方法研究 $[\mathrm{J}]$. 工程力学,2006, 23(8) :42-48.

DING You-liang, LI Ai-qun, MIAO Chang-qing. Investigation on the structural damage alarming method based on wavelet packet energy spectrum $[\mathrm{J}]$. Engineering Mechanics, 2006, 23(8):42-48.

[7] 刘 涛,李爱群,丁幼亮,等. 基于小波包能量谱的结构损 伤预警方法试验研究 $[\mathrm{J}]$. 振动与冲击, 2009,28 (4): $4-8$.

LIU Tao, LI Ai-qun, DING You-liang, et al. Experimental study on structural damage alarming method based on wavelet packet energy spectrum $[\mathrm{J}]$. Journal of Vibration and Shock, $2009,28(4): 4-8$.

[8] 范颖芳,胡志强,周 晶,等. 基于小波包分析的肋拱桥损 伤状态研究 $[\mathrm{J}]$. 工程力学, 2008, 25(5):182-188.

FAN Ying-fang, HU Zhi-qiang, ZHOU Jing, et al. Study on damage state of ribbed arch bridge using wavelet packet analysis [ J ]. Engineering Mechanics, 2008, 25 ( 5): $182-188$

[ 9 ] Sun Z, Chang C C. Structural damage assessment based on 
wavelet packet transform $[\mathrm{J}]$. Journal of Structural Engineering, 2002, 128 (10):1354 - 1361 .

[10] 韩建刚,任伟新,孙增寿. 结构损伤识别的小波包分析试 验研究 $[\mathrm{J}]$. 振动与冲击, 2006, 25(1): 47-50.

HAN Jian-gang, REN Wei-xin, SUN Zeng-shou. Experimental study on structural damage identification based on wavelet packet analysis $[\mathrm{J}]$. Journal of Vibration and Shock, 2006, 25(1): $47-50$.

[11] 姜增国,㫿伟廉,闵志华. 基于小波包分析的结构损伤定 位方法 $[\mathrm{J}]$. 武汉理工大学学报,2006, 28(11):94-97.

JIANG Zeng-guo, QU Wei-lian, MIN Zhi-hua. Damage localization of structures by wavelet packet analysis $[\mathrm{J}]$. Journal of Wuhan University of Technology, 2006, 28 (11) : $94-97$.

[12］袁 颖,周爱红. 结构损伤识别理论及其应用 $[\mathrm{M}]$. 北京: 中国大地出版社, 2008 .

[13] 王振林, 聂国华. 曲率模态和小波包变换在结构损伤识别 中的应用 $[\mathrm{J}]$. 振动与冲击, $2008,27(1): 124-126$.

WANG Zhen-lin, NIE Guo-hua. A method for structure damage defection based on curvature mode and wavelet packet transformation $[\mathrm{J}]$. Journal of Vibration and Shock, 2008, 27 ( 1$): 124-126$.

\section{( 上接第 9 页)}

\section{参 考 文 献}

[ 1 ] 纪洪广, 贾力宏, 李造鼎. 混凝土损伤的声发射模式研究 [J]. 声学学报,1996,21(4):601 -608.

JI Hong-guang, JIA Li-hong, LI Zao-ding. Study on the AEmodel of concrete damage $[\mathrm{J}]$. Acta Acustica, 1996, $21(4): 601-608$.

[2 ] Ohtsu M, Watanabe H. Quantitative damage estimation of concrete by acoustic emission $[\mathrm{J}]$. Construction and Building Materials, 2001, 15(5-6):217 - 224 .

[3] Suzuki T, Ohtsu M. Quantitative damage evaluation of structural concrete by a compression test based on $\mathrm{AE}$ rate process analysis $[\mathrm{J}]$. Construction and Building Materials, 2004, 18(3): $197-202$.

[4] 纪洪广, 蔡美峰. 混凝土材料声发射与应力应变参量耦合 关系及应用 $[\mathrm{J}]$. 岩石力学与工程学报, 2003, 2(2): $227-231$.

JI Hong-guang, CAI Mei-feng. Coupling constitutive relation between $\mathrm{AE}$ parameter and stress-strain and its application $[\mathrm{J}]$. Chinese Journal of Rock Mechanics and Engineering, 2003, 22(2) : $227-231$.

[5] 纪洪广, 张天森, 蔡美峰, 等. 混凝土材料损伤的声发射动 态检测试验研究 $[J]$. 岩石力学与工程学报, $2000,19(2)$ : $165-168$.

JI Hong-guang, ZHANG Tian-sen, CAI Mei-feng, et al. Experimental study on concrete damage by dynamic measurement of acoustic emission $[\mathrm{J}]$. Chinese Journal of Rock Mechanics and Engineering, 2000,19(2) : $165-168$.

[6]朱宏平, 许文胜,陈晓强, 等. 利用声发射信号与速率过程 理论对混凝土损伤进行定量评估 $[\mathrm{J}]$. 工程力学, 2008, 25(1) : $186-191$.

ZHU Hong-ping, XU Wen-sheng, CHEN Xiao-qiang et al. Quantitative concrete-damage evaluation by acoustic emission information and rate-process theory $[\mathrm{J}]$. Engineering Mechanics, 2008 ,25(1): $186-191$.

[ 7 ] Yoon D J, Weiss W J, Shah S P. Assessing damage in corroded reinforced concrete using acoustic emission $[\mathrm{J}]$.
Journal of Engineering Mechanics, 2000, 26(3):273 - 383 .

[ 8 ] Ohstu M, Uchida M, Okamoto T, et al. Damage assessment of reinforced concrete beams qualified by acoustic emission $[\mathrm{J}]$. ACI Structural Journal, 2002, 99(4) : 411 - 417.

[ 9 ] Steven C. Acoustic emission testing of beams to simulate SHM of vintage reinforced concrete deck girder highway bridges [J]. Structure Health Monitoring, 2008 ,7(4) : 329 - 346 .

[10］秦四清, 李造鼎. 岩石声发射参数与断裂力学参量的关系 研究 $[J]$. 东北工学院学报, 1991,12(5):437-444.

QIN Si-qing, LI Zao-ding. On the relationship between rock acoustic emission parameters and fracture mechanics parameters [J ]. Journal of Northeastern University, 1991, 12 ( 5$): 437-444$.

[11] 徐道远, 符晓陵, 祖全民. 混凝土三点弯曲梁断裂能的测试 研究 $[\mathrm{J}]$. 河海大学学报, 1989,17(2):50-56.

XU Dao-yuan, FU Xiao-ling, ZU Quan-min. Fracture energy of three-point bending concrete beams $[\mathrm{J}]$. Journal of Hohai University, 1989, 17(2) : $50-56$.

[12] Guinea G V, Planas J, Elices M. Measurement of the fracture energy using three-point bend tests: part 1 Influence of experimental procedures $[\mathrm{J}]$. Materials and Structures, $1992,25(4): 212-218$.

[13] 党玲博. HRB500 级钢筋混凝土受弯构件裂缝宽度计算研 究 [D]. 郑州: 郑州大学, 2009 .

[14] Barr B I G, Lee M K. Round-robin analysis of the RILEM TC 162-TDF beam-bending test: Part2-approximation of $\delta$ from the CMOD response $[\mathrm{J}]$. Materials and Structures, 2003, $36(9): 621-630$.

[15] Barr B I G, Gettu R. Toughness measurement-the need to think again $[\mathrm{J}]$. Cement and Concrete Composites, 1996, $18(4): 281-297$.

[16] 过镇海, 时旭东. 钢筋混凝土原理和分析 $[\mathrm{M}]$. 北京: 清 华大学出版社, 2009:245-256.

[17 ] Bazant Z P, Oh B H. Crack band theory for fracture of concrete $[\mathrm{J}]$. Materials and Structures, 1983, 16 (3): $155-177$. 